\title{
Fixed point theorems via Generalized WF-Contractions with applications
}

\author{
Rosana Rodríguez-López ${ }^{1}$ (D) $\cdot$ Rakesh Tiwari² $^{2}$
}

Received: 19 July 2020 / Accepted: 22 July 2021 / Published online: 21 August 2021

(c) The Author(s) 2021

\begin{abstract}
The aim of this paper is to introduce a new class of mixed contractions which allow to revise and generalize some results obtained in [6] by R. Gubran, W. M. Alfaqih and M. Imdad. We also provide an example corresponding to this class of mappings and show how the new fixed point result relates to the above-mentioned result in [6]. Further, we present an application to the solvability of a two-point boundary value problem for second order differential equations.
\end{abstract}

Keywords Fixed points · WF-Contractions · Generalized WF-Contractions

Mathematics Subject Classification 54H25 · 47H10

\section{Introduction}

Fixed point theory is a relevant area of research, mainly due to its applicability to various fields, specially in the study of the properties of the solutions to differential equations. Banach Contraction Principle is the most celebrated result, and it provides not only the existence, but also the uniqueness and an iterative process to approximate the fixed point. This theorem has been generalized in various ways, including its extension to partially ordered sets $[9,10,14]$. Another relevant extension was given by Alber and Guerre-Delabriere for weakly contractive maps in Hilbert Spaces, see [1]. Rhoades [15] continued the study on the topic and also Dutta and Chaudhury [5] provided a generalization. The starting point in our study will be the result by Gubran, Alfaqih and Imdad included in [6], where the following terminology was used.

Rosana Rodríguez-López

rosana.rodriguez.lopez@usc.es

Rakesh Tiwari

rtiwari@govtsciencecollegedurg.ac.in

1 Instituto de Matemáticas, Departamento de Estatística, Análise Matemática e Optimización, Facultade de Matemáticas, Universidade de Santiago de Compostela, 15782 Santiago de Compostela, Spain

2 Department of Mathematics, Government V. Y. T. Post-Graduate Autonomous College, Chhattisgarh, Durg 491001, India 
Similar to [6], we denote by $\Psi$ the set of all continuous and monotonic nondecreasing functions $\psi:[0, \infty) \rightarrow[0, \infty)$ such that

$$
\psi(t)=0 \text { if and only if } t=0 .
$$

They recall the following uniqueness result from [5], where a weak contractivity condition is considered by using functions in the set $\Psi$.

Theorem 1 [5] Let $(X, d)$ be a complete metric space and $f: X \rightarrow X$ be a weak contractive mapping, that is, a mapping satisfying

$$
\psi(d(f x, f y)) \leq \psi(d(x, y))-\varphi(d(x, y)), \text { for all } x, y \in X,
$$

where $\psi, \varphi \in \Psi$. Then $f$ has a unique fixed point.

Some other interesting concepts to deal with weak contractions are, for instance, the notion of implicit contractive function given in 1997 by Popa [12], which was later discussed and considered by other authors, as we can see, for instance, in the research works $[2,3,7,8,13]$. Recently, Tiwari and Gupta [16] proved some new common fixed point theorems in metric spaces for weakly compatible mappings satisfying an implicit relation involving quadratic terms. Another interesting work is that by Wardowski [17], who gave in 2012 an extension of Contraction Mapping Principle for the case of the new concept of F-contractions, notion that fits adequately in the sense that the corresponding mappings exhibit uniqueness of fixed point in the context of complete metric spaces.

\section{Preliminaries}

We begin by introducing some concepts needed, where we denote $\mathbb{R}_{+}=[0, \infty)$. The notion of $F$-contraction introduced by Wardowski is presented in Definition 1. For this definition, the author considered the class of functions $\mathfrak{F}$ consisting of all functions $F: \mathbb{R}_{+} \rightarrow \mathbb{R}$ satisfying the conditions $(\mathbf{F 1})-(\mathbf{F 3})$ specified below:

(F1): $F$ is strictly increasing.

(F2): For every sequence $\left\{s_{n}\right\}$ of positive real numbers, we have

$$
\lim _{n \rightarrow \infty} s_{n}=0 \Longleftrightarrow \lim _{n \rightarrow \infty} F\left(s_{n}\right)=-\infty .
$$

(F3): There exists $k \in(0,1)$ such that $\lim _{s \rightarrow 0^{+}} s^{k} F(s)=0$.

As mentioned in [6], the functions $F$ given, respectively, by $F(s)=\ln (s), F(s)=s+\ln (s)$, and $F(s)=\frac{-1}{\sqrt{s}}$, belong to the family $\mathfrak{F}$.

Definition 1 ([17]) We say that a self-mapping $f$ on a metric space $(X, d)$ is an F-contraction if there exists $\tau \geq 0$ such that, for all $x, y \in X$ with $d(f x, f y)>0$, we have

$$
\tau+F(d(f x, f y)) \leq F(d(x, y)),
$$

where $F: \mathbb{R}_{+} \rightarrow \mathbb{R}$ is a mapping satisfying conditions (F1)-(F3).

More recently, in [6], Gubran et al. introduced a new class of contractions, called WFcontractions, presented as a mixed type of weak and F-contractions, but different from both concepts, and defined as explained in Definition 2.

For this concept, they considered two families of functions: 
$\mathbb{G}$ The family $\mathbb{G}$ given by all functions $G:[0, \infty) \rightarrow[0, \infty)$ satisfying the following two properties:

(G1): $G$ is strictly increasing.

(G2)*: There exists $k \in(0,1)$ such that $\lim _{s \rightarrow 0^{+}} s^{k} G(s)=0$.

$\Delta$ The family $\Delta$ given by all functions $\delta:[0, \infty) \rightarrow[0, \infty)$ satisfying the property: $(\mathbf{G 3})^{*}: \delta(t)>0$ for all $t>0$ and, for every strictly decreasing sequence $\left\{s_{n}\right\}$ of positive real numbers, we have

$$
\lim _{n \rightarrow \infty} \delta\left(s_{n}\right)=0 \Rightarrow \lim _{n \rightarrow \infty} s_{n}=0 .
$$

Definition 2 [6] A self-mapping $f$ on a metric space $(X, d)$ is said to be a $W F$-contraction if there exist two functions $G, \delta:[0, \infty) \rightarrow[0, \infty)$ such that, for all $x, y \in X$ with $d(f x, f y)>0$, we have

$$
\delta(d(x, y))+G(d(f x, f y)) \leq G(d(x, y)),
$$

where $G \in \mathbb{G}$ and $\delta \in \Delta$.

The authors of [6] proved some fixed point results for this class of mappings, as we recall below.

Lemma 1 [6, Lemma 2.1] A WF-contraction has at most one fixed point.

Lemma 2 [6, Lemma 2.2] Let $(X, d)$ be a metric space and $\left\{t_{n}\right\}$ be a sequence of positive real numbers such that

$$
\delta\left(t_{n}\right)+G\left(t_{n+1}\right) \leq G\left(t_{n}\right),
$$

for all $n$, where $G \in \mathbb{G}$ and $\delta \in \Delta$. Then, the sequence $\left\{t_{n}\right\}$ is strictly decreasing and

$$
\sum_{i=1}^{\infty} \delta\left(t_{i}\right)<\infty .
$$

Theorem 2 [6, Theorem 2.1] Let $(X, d)$ be a complete metric space and consider $f: X \rightarrow X$ $a$ WF-contraction for some $G \in \mathbb{G}$ and $\delta \in \Delta$. Then, $f$ has a unique fixed point.

The authors in [6] also justified that the concept of WF-contraction is different from the notion of weak contraction, and that each WF-contraction $f$ satisfies $d(f x, f y)<d(x, y)$, for $x, y$ different, so that the continuity of the mapping is guaranteed.

However, in the proof of [6, Theorem 2.1], some confusing points have been detected in the following sense. To prove that a particular sequence $\left\{x_{n}\right\}$, which is understood to converge to a fixed point of the mapping, is a Cauchy sequence, it is considered $M:=\min _{0 \leq i \leq n} \delta\left(t_{i}\right)$ and then deduced that

$$
\lim _{n \rightarrow \infty}(n+1) t_{n+1}^{k} M=0
$$

where $t_{n}=d\left(x_{n}, x_{n+1}\right) \rightarrow 0$ as $n \rightarrow 0$, and hence argued that it is possible to find $N \in \mathbb{N}$ such that $n t_{n}^{k} \leq 1$ for all $n \geq N$. However, $M$ should be a sequence instead, that is,

$$
M_{n}:=\min _{0 \leq i \leq n} \delta\left(t_{i}\right)
$$


satisfying $\lim _{n \rightarrow \infty}(n+1) t_{n+1}^{k} M_{n}=0$, and $M_{n}$ could tend to zero, so it is not possible to deduce that $n t_{n}^{k} \leq 1$ for $n$ large enough.

The purpose of this paper is to revise some of these aspects and to introduce a new class of mixed contractions in order to generalize this result by Gubran et al. proved in [6], giving a more general class of mappings which allow to deduce the existence of a unique fixed point. We also show an example of a mapping in this class. Further, we show an application to the solvability of a two-point boundary value problem for second-order differential equations.

\section{Main result}

In this section, we introduce the concept of a generalized WF-contraction, and establish a fixed point theorem valid for complete metric spaces.

We consider two families of functions:

$\widetilde{\mathbb{G}}$ The family $\widetilde{\mathbb{G}}$ given by all functions $G:[0, \infty) \rightarrow[0, \infty)$ satisfying the following property:

(G1): $G$ is strictly increasing.

$\widetilde{\Delta}$ The family $\widetilde{\Delta}$ given by all functions $\delta:[0, \infty) \rightarrow[0, \infty)$ satisfying the following two properties:

(G2): $\delta(t)>0$ for all $t>0$ and, for every strictly decreasing sequence $\left\{s_{n}\right\}$ of positive real numbers, we have

$$
\lim _{n \rightarrow \infty} \delta\left(s_{n}\right)=0 \Rightarrow \lim _{n \rightarrow \infty} s_{n}=0 .
$$

(G3): For every strictly decreasing and convergent to zero sequence $\left\{s_{n}\right\}$ of positive real numbers, we have

$$
\sum_{n=0}^{\infty} \delta\left(s_{n}\right)<\infty \Rightarrow \sum_{n=0}^{\infty} s_{n}<\infty .
$$

Definition 3 We say that a self-mapping $f$ on a metric space $(X, d)$ is a Generalized WFcontraction if there exist two functions $G, \delta:[0, \infty) \rightarrow[0, \infty)$ such that, for all $x, y \in X$ with $d(f x, f y)>0$, we have

$$
\delta(d(x, y))+G(d(f x, f y)) \leq G(\max \{d(x, y), d(x, f x), d(y, f y)\}),
$$

where $G$ satisfies condition $(\mathbf{G 1})$, and $\delta$ satisfies the conditions $(\mathbf{G 2})$ and $(\mathbf{G 3})$, that is, $G \in \widetilde{\mathbb{G}}$ and $\delta \in \widetilde{\Delta}$.

Next, we give an example of mapping satisfying condition (4), which is similar to the one proposed in [6].

Example 1 We consider the base space of nonnegative real numbers $X=[0, \infty)$ and define a self mapping $f$ on $X$ by

$$
f(x)=\left\{\begin{array}{ll}
\frac{x+6}{3}, & \text { if } x \leq 3, \\
3, & \text { if } x \geq 3 .
\end{array} \text { Then the mapping } f\right. \text { satisfies (4), taking the func- }
$$

tions $G(s)=s+\frac{1}{3(s+1)}$ and $\delta(t)=\frac{t}{9}$, for which it is obvious the validity of conditions (G1)-(G3). 
Indeed, we distinguish three cases:

Case I. If $3 \geq y \geq x$, then the inequality (4) is written as:

$$
\begin{aligned}
\frac{y-x}{9}+\frac{y-x}{3}+\frac{1}{3\left(\frac{y-x}{3}+1\right)} & \leq G\left(\max \left\{y-x, \frac{x+6}{3}-x, \frac{y+6}{3}-y\right\}\right) \\
& =G\left(\max \left\{y-x, \frac{2}{3}(3-x), \frac{2}{3}(3-y)\right\}\right) \\
& =G\left(\max \left\{y-x, \frac{2}{3}(3-x)\right\}\right) .
\end{aligned}
$$

If $\frac{2}{3}(3-x) \leq y-x$, we have

$$
\frac{y-x}{9}+\frac{y-x}{3}+\frac{1}{3\left(\frac{y-x}{3}+1\right)} \leq G(y-x),
$$

which yields

$$
\frac{4(y-x)}{9}+\frac{1}{3\left(\frac{y-x}{3}+1\right)} \leq(y-x)+\frac{1}{3(y-x+1)} .
$$

This can be written as

$$
0 \leq \frac{5 z}{9}+\frac{1}{3(z+1)}-\frac{1}{z+3}
$$

by defining $z:=y-x \geq 0$. The right-hand side in (5) represents a mapping of the variable $z$ which is increasing on $[0,3]$ and vanishing at $z=0$.

On the other hand, if $\frac{2}{3}(3-x)>y-x$, we have

$$
\frac{y-x}{9}+\frac{y-x}{3}+\frac{1}{3\left(\frac{y-x}{3}+1\right)} \leq G\left(\frac{2}{3}(3-x)\right),
$$

that is,

$$
\frac{4(y-x)}{9}+\frac{1}{3\left(\frac{y-x}{3}+1\right)} \leq \frac{2}{3}(3-x)+\frac{1}{3\left(\frac{2}{3}(3-x)+1\right)} .
$$

This can be written as

$$
\frac{4 y}{9}+\frac{1}{y-x+3} \leq 2-\frac{2}{9} x+\frac{1}{9-2 x} .
$$

Note that, since $y-x \geq 0$ and $y<2+\frac{1}{3} x$,

$$
\frac{4 y}{9}+\frac{1}{y-x+3} \leq \frac{4}{9}\left(2+\frac{1}{3} x\right)+\frac{1}{3}=\frac{11}{9}+\frac{4}{27} x \leq 2-\frac{2}{9} x+\frac{1}{9-2 x} .
$$

The last inequality is valid on the region considered since

$$
\gamma(x):=2-\frac{2}{9} x+\frac{1}{9-2 x}-\frac{11}{9}-\frac{4}{27} x=\frac{7}{9}-\frac{10}{27} x+\frac{1}{9-2 x}
$$

has derivative

$$
\gamma^{\prime}(x)=-\frac{10}{27}+\frac{2}{(9-2 x)^{2}},
$$


which is negative on [0,3], and $\gamma(3):=\frac{7}{9}-3 \frac{10}{27}+\frac{1}{3}=0$.

Case II. If $y \geq 3 \geq x$, then (4) is expressed as:

$$
\frac{y-x}{9}+G\left(3-\frac{x+6}{3}\right) \leq G\left(\max \left\{y-x, \frac{x+6}{3}-x, y-3\right\}\right) \text {. }
$$

Since $y-x \geq y-3$, and $\frac{x+6}{3} \leq \frac{3+6}{3}=3 \leq y$, we have

$$
\frac{y-x}{9}+G\left(\frac{3-x}{3}\right) \leq G(y-x),
$$

or

$$
\frac{y-x}{9}+\frac{3-x}{3}+\frac{1}{3\left(\frac{3-x}{3}+1\right)} \leq(y-x)+\frac{1}{3(y-x+1)},
$$

that is,

$$
\frac{3-x}{3}+\frac{1}{6-x} \leq \frac{8}{9}(y-x)+\frac{1}{3(y-x+1)} .
$$

Similar to the Example in [6], we denote $a:=3-x$ and $b:=y-3$. Thus, the previous inequality is written as:

$$
\frac{a}{3}+\frac{1}{3+a} \leq \frac{8}{9}(a+b)+\frac{1}{3(a+b+1)}
$$

or, equivalently,

$$
\frac{2 a+3 b}{3(3+a)(a+b+1)} \leq \frac{5 a+8 b}{9},
$$

which is obviously true.

Case III. If $3 \leq x \leq y$, then nothing has to be checked since

$$
d(f x, f y)=|3-3|=0 .
$$

In any case, the inequality in (4) would be valid since it reduces to:

$$
\frac{y-x}{9}+\frac{1}{3} \leq G(\max \{y-x, x-3, y-3\})=G(y-3),
$$

or

$$
\frac{y-x}{9}+\frac{1}{3} \leq(y-3)+\frac{1}{3(y-3+1)},
$$

which can be expressed as:

$$
-\frac{x}{9}+\frac{1}{3} \leq \frac{8}{9} y-3+\frac{1}{3(y-2)} .
$$

Since $x \geq 3$,

$$
-\frac{x}{9}+\frac{1}{3} \leq 0 \leq \frac{8}{9} y-3+\frac{1}{3(y-2)},
$$

and the last inequality holds because $\gamma(y)=\frac{8}{9} y-3+\frac{1}{3(y-2)}$ is such that $\gamma(3)=\frac{8}{3}-3+\frac{1}{3}=0$ and $\gamma^{\prime}(y)=\frac{8}{9}+\frac{-1}{9(y-2)^{2}}>0$ on $[3,+\infty)$. 
Similar to the result for WF-contractions, we deduce the uniqueness of fixed point (provided it exists).

Lemma 3 A Generalized WF-contraction has at most one fixed point.

Proof Let $x, y \in X$ be two different fixed points of $f$, then, from (4), we have

$$
\delta(d(x, y))+G(d(x, y)) \leq G(\max \{d(x, y), d(x, x), d(y, y)\}),
$$

which gives $\delta(d(x, y)) \leq 0$, a contradiction due to the fact that $\delta(t)>0$ for $t \in(0, \infty)$.

Remark 3 Since it will be used later, we explain the hypotheses required on functions $G$ and $\delta$ for the validity of Lemma 2 [6, Lemma 2.2]. By revising the proof in [6], we find that the conclusion holds just by assuming that $G$ is strictly increasing and $\delta(t)>0$ for $t \in(0, \infty)$.

Next, we provide the result on the existence of a unique fixed point for the mapping $f$. We use the notation $\mathbb{N}_{0}:=\mathbb{N} \cup\{0\}$.

Theorem 4 Consider a complete metric space $(X, d)$ and suppose that the mapping $f: X \rightarrow$ $X$ is continuous and satisfies the Generalized WF-contraction property for some $G \in \widetilde{\mathbb{G}}$ and $\delta \in \widetilde{\Delta}$. Then, there exists a unique fixed point for the mapping $f$.

Proof We start by selecting an arbitrary element $x_{0} \in X$, and defining the sequence $\left\{x_{n}\right\}$ in $X$ by recurrence, as follows:

$$
x_{n+1}:=f x_{n}, \text { for all } n \in \mathbb{N}_{0} .
$$

In case that $x_{n}=x_{n+1}$ for some $n \in \mathbb{N}_{0}$, then we have proved the existence of a fixed point for $f$. Therefore, we assume that $d\left(x_{n}, x_{n+1}\right)>0$ for every $n \in \mathbb{N}_{0}$. Similar to [6], we denote by $t_{n}:=d\left(x_{n}, x_{n+1}\right), n \in \mathbb{N}_{0}$, so that $\left\{t_{n}\right\}$ is a sequence of positive real numbers. Now, choosing $x=x_{n}$ and $y=x_{n+1}$ in the inequality (4), we get

$$
\delta\left(t_{n}\right)+G\left(t_{n+1}\right) \leq G\left(\max \left\{t_{n}, t_{n+1}\right\}\right) .
$$

By the positiveness of $\delta$ on $(0,+\infty)$ and the strictly increasing character of $G$, we get that

$$
G\left(t_{n+1}\right)<G\left(\max \left\{t_{n}, t_{n+1}\right\}\right)=\max \left\{G\left(t_{n}\right), G\left(t_{n+1}\right)\right\},
$$

so that

$$
\max \left\{G\left(t_{n}\right), G\left(t_{n+1}\right)\right\}=G\left(t_{n}\right),
$$

and (7) is reduced to

$$
\delta\left(t_{n}\right)+G\left(t_{n+1}\right) \leq G\left(t_{n}\right),
$$

for every $n \in \mathbb{N}_{0}$. Hence, similar to Theorem 2.1 [6], by applying Lemma 2 (see Remark 3), we deduce the strictly decreasing character of the sequence $\left\{t_{n}\right\}$ and the convergence of the series $\sum_{i=0}^{\infty} \delta\left(t_{i}\right)<\infty$, thus, the general term is convergent to zero, that is, $\lim _{n \rightarrow \infty} \delta\left(t_{n}\right)=0$, which implies that

$$
\lim _{n \rightarrow \infty} t_{n}=0
$$

by virtue of condition (G2). By (G3), and the convergence of $\sum_{i=0}^{\infty} \delta\left(t_{i}\right)<\infty$, we deduce the convergence of $\sum_{i=0}^{\infty} t_{i}<\infty$. 
Now, we prove that $\left\{x_{n}\right\}$ is a Cauchy sequence. Let $m, n \in \mathbb{N}$ with $m>n$, then we obtain

$$
d\left(x_{m}, x_{n}\right) \leq \sum_{i=n}^{m-1} t_{i}<\sum_{i=n}^{\infty} t_{i} \rightarrow 0, \text { as } n \rightarrow \infty,
$$

due to the convergence of $\sum_{i=0}^{\infty} t_{i}<\infty$. This proves that $\left\{x_{n}\right\}$ is a Cauchy sequence.

By the completeness of $X$, there exists $x \in X$ which is its limit, and using the continuity of $f$, we have

$$
x=\lim _{n \rightarrow \infty} x_{n+1}=f\left(\lim _{n \rightarrow \infty} x_{n}\right)=f x .
$$

Since the uniqueness was justified in Lemma 3, the proof is finished.

Remark 5 In the statement and proof of Theorem 4, the family $\widetilde{\Delta}$ can be replaced by:

$\widehat{\Delta}$ The family $\widehat{\Delta}$ given by all functions $\delta:[0, \infty) \rightarrow[0, \infty)$ satisfying the following two properties:

$(\widehat{\mathbf{G 2}}): \delta(t)>0$ for all $t>0$.

$(\widehat{\mathbf{G 3}})$ : For every strictly decreasing sequence $\left\{s_{n}\right\}$ of positive real numbers, we have

$$
\sum_{n=0}^{\infty} \delta\left(s_{n}\right)<\infty \Rightarrow \sum_{n=0}^{\infty} s_{n}<\infty
$$

Lemma 4 Consider:

$\bar{\Delta}$ The family $\bar{\Delta}$ of all functions $\delta:[0, \infty) \rightarrow[0, \infty)$ satisfying the following three properties:

$(\widehat{\mathbf{G 2}}): \delta(t)>0$ for all $t>0$.

$(\overline{\mathbf{G 3}})$ : If $t, \mathrm{~s}$ are positive real numbers, then

$$
\delta(t+s) \leq \delta(t)+\delta(s) .
$$

$(\overline{\mathbf{G 4}})$ : For every strictly increasing sequence $\left\{z_{n}\right\}$ of positive real numbers, we have

$$
\text { if }\left\{\delta\left(z_{n}\right)\right\} \text { is bounded } \Rightarrow\left\{z_{n}\right\} \text { is bounded. }
$$

Then $\bar{\Delta} \subseteq \widehat{\Delta}$.

Proof We prove that $\bar{\Delta} \subseteq \widehat{\Delta}$ by checking that conditions $(\overline{\mathbf{G 3}})-(\overline{\mathbf{G 4}})$ imply $(\widehat{\mathbf{G 3}})$. Indeed, let $\left\{s_{n}\right\}$ be a strictly decreasing sequence of positive real numbers, and assume that:

$$
\sum_{n=0}^{\infty} \delta\left(s_{n}\right)<\infty
$$

Consider the convergent sequence of partial sums $\left\{y_{n}\right\}$, where $y_{n}=\sum_{j=0}^{n} \delta\left(s_{j}\right)$, and the strictly increasing sequence $\left\{z_{n}\right\}$ of positive real numbers given by $z_{n}=\sum_{j=0}^{n} s_{j}$. Then, by $(\overline{\mathbf{G 3}})$,

$$
0<\delta\left(z_{n}\right)=\delta\left(\sum_{j=0}^{n} s_{j}\right) \leq \sum_{j=0}^{n} \delta\left(s_{j}\right)=y_{n}, \text { for all } n \in \mathbb{N}
$$


Since $\left\{y_{n}\right\}$ is convergent, then it is bounded, so $\left\{\delta\left(z_{n}\right)\right\}$ is a bounded sequence. By $(\overline{\mathbf{G 4}}),\left\{z_{n}\right\}$ is also bounded. Moreover, it is an increasing sequence, so $\left\{z_{n}\right\}$ is convergent, but this is the sequence of partial sums of the series $\sum_{n=0}^{\infty} s_{n}$, whose sum is finite.

Remark 6 It is clear that a WF-contraction is continuous and also satisfies the inequality required to be a Generalized WF-contraction (by the nondecreasing character of $G$ ), so Theorem 4 provides a generalized inequality in comparison to Theorem 2.1 [6]. However, we have modified the families $\mathbb{G}$ (extending it) and $\Delta$ (adding or modifying the conditions), in order to clarify the procedure.

In the following Corollary, we consider the particular case of $G(t)=t$, and $\delta(t)=\mu t$, where $\mu>0$, which belong to the families $\mathbb{G}$ and $\widetilde{\Delta} \cap \widehat{\Delta}$, respectively.

Corollary 7 Consider a complete metric space $(X, d)$ and suppose that the mapping $f$ : $X \rightarrow X$ is continuous and that, for all $x, y \in X$ with $d(f x, f y)>0$, we have

$$
d(f x, f y) \leq \max \{d(x, y), d(x, f x), d(y, f y)\}-\mu d(x, y) .
$$

Then, there exists a unique fixed point for the mapping $f$.

Last result is an extension of Banach Contraction Principle since a function satisfying

$$
d(f x, f y) \leq c d(x, y), \text { for all } x, y \in X,
$$

with $c \in[0,1)$, is continuous and satisfies

$$
\begin{aligned}
d(f x, f y) & \leq(1-(1-c)) d(x, y) \\
& \leq \max \{d(x, y), d(x, f x), d(y, f y)\}-\mu d(x, y),
\end{aligned}
$$

for all $x, y \in X$, where $\mu:=1-c \in(0,1]$.

Also, by taking $G(t)=\ln (t+r)$, where $r$ is fixed with $r \in(0,1]$ and $\delta \in \widetilde{\Delta} \cup \widehat{\Delta}$, we have the following Corollary.

Corollary 8 Suppose that $r$ is fixed with $r \in(0,1]$ and that $\delta \in \widetilde{\Delta} \cup \widehat{\Delta}$. Consider a complete metric space $(X, d)$ and suppose that the mapping $f: X \rightarrow X$ is continuous and that, for all $x, y \in X$ with $d(f x, f y)>0$, we have

$$
d(f x, f y) \leq e^{-\delta(d(x, y))}[\max \{d(x, y), d(x, f x), d(y, f y)\}+r]-r .
$$

Then, there exists a unique fixed point for the mapping $f$.

\section{Application}

Similar to the application shown in [6], we present an example of a two-point boundary value problem for differential equations of second order, and we apply the new results in order to deduce the existence of a unique solution. The problem considered is similar to equation (4.1) in [6]:

$$
\left\{\begin{array}{l}
-x^{\prime \prime}(t)=g(t, x(t)), \quad t \in J=[0,1], \\
x(0)=\alpha, x(1)=\beta,
\end{array}\right.
$$

where $\alpha, \beta \in \mathbb{R}$, and $g: J \times \mathbb{R} \rightarrow \mathbb{R}$ is a continuous function. For the above-mentioned boundary value problem (13), the Green's function is defined as:

$$
K(t, s)= \begin{cases}s(1-t), & 0 \leq s<t \leq 1 \\ t(1-s), & 0 \leq t \leq s \leq 1\end{cases}
$$


Considering the space $\mathcal{C}(J)$ consisting of all continuous real functions defined on $J$, it is well-known that $\left(\mathcal{C}(J), d_{\theta}\right)$ is a complete metric space if we consider the weighted supremum distance

$$
d_{\theta}(x, y)=\max _{t \in J}\left\{|x(t)-y(t)| e^{-\theta t}\right\}, \quad x, y \in \mathcal{C}(J),
$$

where $\theta \geq 0$ is fixed. In the following, we use $d:=d_{0}$ the supremum norm.

By applying the results proved, we can deduce the existence of a unique solution for problem (13).

Theorem 9 Suppose that there exists $\delta \in \widetilde{\Delta} \cup \widehat{\Delta}$ such that, for all $t, s \in J$ and non-identical $x, y \in \mathcal{C}(J)$, we have

$$
\begin{aligned}
& |K(t, s)[g(s, x(s))-g(s, y(s))]| \\
& \leq \max \{|x(s)-y(s)|, \\
& \quad\left|x(s)-\alpha-(\beta-\alpha) t-\int_{0}^{1} K(t, s) g(s, x(s)) d s\right|, \\
& \left.\quad\left|y(s)-\alpha-(\beta-\alpha) t-\int_{0}^{1} K(t, s) g(s, y(s)) d s\right|\right\} \\
& -\delta(d(x, y)) .
\end{aligned}
$$

Then, problem (13) has a unique solution $x^{*} \in \mathcal{C}^{2}$.

Proof It is well known that $x \in \mathcal{C}^{2}$ is a solution to (13) if and only if $x \in \mathcal{C}$ is a solution to

$$
x(t)=\alpha+(\beta-\alpha) t+\int_{0}^{1} K(t, s) g(s, x(s)) d s, \text { for all } \mathrm{t} \in J .
$$

Hence, we can define a mapping $f: \mathcal{C}(J) \rightarrow \mathcal{C}(J)$ by

$$
f x(t)=\alpha+(\beta-\alpha) t+\int_{0}^{1} K(t, s) g(s, x(s)) d s, \text { for all } \mathrm{t} \in J .
$$

This definition of $f$ clearly allows to affirm that the fixed points of $f$ in $\mathcal{C}(J)$ are the continuous solutions to (15), and, therefore, the solutions to the boundary value problem (13).

Next, we check the validity of the conditions in Theorem 4. It is obvious that $f$ is a continuous mapping. Let $x, y \in \mathcal{C}(J)$ with $d(f x, f y)>0$, that is, $x$ not coincident with $y$, then we obtain, for $t \in J$, that

$$
\begin{aligned}
& |f x(t)-f y(t)|=\left|\int_{0}^{1} K(t, s) g(s, x(s)) d s-\int_{0}^{1} K(t, s) g(s, y(s)) d s\right| \\
& \leq \int_{0}^{1}|K(t, s)[g(s, x(s))-g(s, y(s))]| d s \\
& \leq \int_{0}^{1}\left(\operatorname { m a x } \left\{|x(s)-y(s)|,\left|x(s)-\alpha-(\beta-\alpha) t-\int_{0}^{1} K(t, s) g(s, x(s)) d s\right|,\right.\right. \\
& \left.\left.\quad\left|y(s)-\alpha-(\beta-\alpha) t-\int_{0}^{1} K(t, s) g(s, y(s)) d s\right|\right\}-\delta(d(x, y))\right) d s \\
& \leq \max \{d(x, y), d(x, f x), d(y, f y)\}-\delta(d(x, y)),
\end{aligned}
$$

therefore

$$
d(f x, f y)) \leq \max \{d(x, y), d(x, f x), d(y, f y)\}-\delta(d(x, y)) .
$$


This proves that condition (4) is satisfied for $G$ chosen as the identity mapping. By applying Theorem 4, there exists a unique solution to problem (13).

We can extend Theorem 9 in the following way.

Theorem 10 Suppose that there exist $G \in \mathbb{G}$, and $\delta \in \widetilde{\Delta} \cup \widehat{\Delta}$ such that, for all $t, s \in J$ and non-identical $x, y \in \mathcal{C}(J)$, we have

$$
\begin{aligned}
& |K(t, s)[g(s, x(s))-g(s, y(s))]| \\
& \leq G^{-1}[G(\max \{|x(s)-y(s)|, \\
& \quad\left|x(s)-\alpha-(\beta-\alpha) t-\int_{0}^{1} K(t, s) g(s, x(s)) d s\right|, \\
& \left.\left.\quad\left|y(s)-\alpha-(\beta-\alpha) t-\int_{0}^{1} K(t, s) g(s, y(s)) d s\right|\right\}\right) \\
& \quad-\delta(d(x, y))] .
\end{aligned}
$$

Then, problem (13) has a unique solution $x^{*} \in \mathcal{C}^{2}$.

Proof It is obvious since both $G$ and $G^{-1}$ are strictly increasing functions.

Acknowledgements The research of R. Rodríguez-López is partially supported by grant numbers MTM201675140-P (AEI/FEDER, UE) and ED431C 2019/02 (GRC Xunta de Galicia).

The authors are grateful to the Editor in Chief and the anonymous Referees for their helpful comments towards the improvement of the paper.

Funding Open Access funding provided thanks to the CRUE-CSIC agreement with Springer Nature.

Open Access This article is licensed under a Creative Commons Attribution 4.0 International License, which permits use, sharing, adaptation, distribution and reproduction in any medium or format, as long as you give appropriate credit to the original author(s) and the source, provide a link to the Creative Commons licence, and indicate if changes were made. The images or other third party material in this article are included in the article's Creative Commons licence, unless indicated otherwise in a credit line to the material. If material is not included in the article's Creative Commons licence and your intended use is not permitted by statutory regulation or exceeds the permitted use, you will need to obtain permission directly from the copyright holder. To view a copy of this licence, visit http://creativecommons.org/licenses/by/4.0/.

\section{References}

1. Alber, Y. I., Guerre-Delabriere, S.: Principle of weakly contractive maps in Hilbert spaces, in: New Results in Operator Theory and Its Applications, Springer-Verlag, Basel, 7-22 (1997)

2. Ali, J., Imdad, M.: An implicit function implies several contraction conditions. Sarajevo J. Math. 4, 269-285 (2008)

3. Altun, I., Turkoglu, D.: Some fixed point theorems for weakly compatible mappings satisfying an implicit relation. Taiwanese J. Math. 13, 1291-1304 (2009)

4. Argoubi, H., Samet, B., Vetro, C.: Nonlinear contractions involving simulation functions in a metric space with a partial order. J. Nonlinear Sci. Appl. 8, 1082-1094 (2015)

5. Dutta, P., Choudhury, B. S.: A generalization of contraction principle in metric spaces, Fixed Point Theory Appl. 2008, Article ID 406368, 8 pages (2008)

6. Gubran, R., Alfaqih, W.M., Imdad, M.: Fixed point theorems via WF-contractions. Kragujevac J. Math. 45(3), 353-360 (2021)

7. Imdad, M., Ali, J.: A general fixed point theorem in fuzzy metric spaces via an implicit function. J. Appl. Math. Inform. 26, 591-603 (2008)

8. Imdad, M., Gubran, R., Ahmadullah, M.: Using an implicit function to prove common fixed point theorems. J. Adv. Math. Stud. 11(3), 481-491 (2018) 
9. Nieto, J.J., Rodríguez-López, R.: Contractive mapping theorems in partially ordered sets and applications to ordinary differential equations. Order 22(3), 223-239 (2005)

10. Nieto, J.J., Rodríguez-López, R.: Existence and uniqueness of fixed point in partially ordered sets and applications to ordinary differential equations. Acta Mathematica Sinica, English Series 23(12), 22052212 (2007)

11. O'Regan, D., Petrusel, A.: Fixed point theorems for generalized contractions in ordered metric spaces. J. Math. Anal. Appl. 341, 1241-1252 (2008)

12. Popa, V.: Fixed point theorems for implicit contractive mappings. Stud. Cerc. St. Ser. Mat. Univ. Bacau 7, 127-133 (1997)

13. Popa, V.: A general fixed point theorem for weakly compatible mappings in compact metric spaces. Turkish J. Math. 25, 465-474 (2001)

14. Ran, A.C.M., Reurings, M.C.B.: A fixed point theorem in partially ordered sets and some applications to matrix equations. Proc. Amer. Math. Soc. 132, 1435-1443 (2004)

15. Rhoades, B.: Some theorems for weakly contractive maps. Nonlinear Anal. 47, 2683-2693 (2001)

16. Tiwari, R., Gupta, S.: Some common fixed point theorems in metric spaces satisfying an implicit relation involving quadratic terms. Funct. Anal. Approxim. Compt. 8(2), 45-51 (2016)

17. Wardowski, D.: Fixed points of a new type of contractive mappings in complete metric spaces, Fixed Point Theory Appl. Article ID 94, 6 pages (2012)

Publisher's Note Springer Nature remains neutral with regard to jurisdictional claims in published maps and institutional affiliations. 